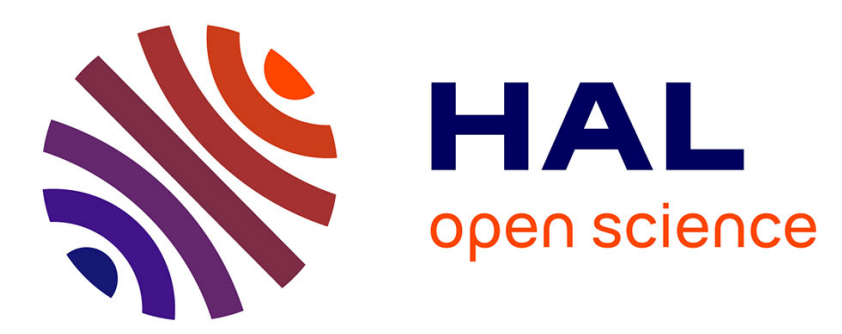

\title{
Robust GrayScale Distribution Estimation for Contactless Palmprint Recognition
}

\author{
Julien Doublet, Marinette Revenu, Olivier Lepetit
}

\section{To cite this version:}

Julien Doublet, Marinette Revenu, Olivier Lepetit. Robust GrayScale Distribution Estimation for Contactless Palmprint Recognition. IEEE Conference on biometrics : theory, applications and systems, 2007, Washington, United States. 6 p, 10.1109/BTAS.2007.4401935 . hal-00259521

\section{HAL Id: hal-00259521 \\ https://hal.science/hal-00259521}

Submitted on 20 Oct 2008

HAL is a multi-disciplinary open access archive for the deposit and dissemination of scientific research documents, whether they are published or not. The documents may come from teaching and research institutions in France or abroad, or from public or private research centers.
L'archive ouverte pluridisciplinaire HAL, est destinée au dépôt et à la diffusion de documents scientifiques de niveau recherche, publiés ou non, émanant des établissements d'enseignement et de recherche français ou étrangers, des laboratoires publics ou privés. 


\title{
Robust GrayScale Distribution Estimation for Contactless Palmprint Recognition
}

\author{
Julien Doublet, Marinette Revenu and Olivier Lepetit
}

\begin{abstract}
More and more research have been developed very recently for automatic hand recognition. This paper proposes a new method for contactless hand authentication in complex images. Our system uses skin color and hand shape information for an accurate hand detection process. Then, the palm is extracted and characterized by a robust and normalized decomposition. During enrollment, a distribution estimation is used to defined the optimal discrimination of the palmprint features. Finally, some specific thresholds are defined to separate in test phase impostor and genuine users. The experimental results present an error rate of $1.5 \%$ with a population of 49 people.
\end{abstract}

\section{INTRODUCTION}

Recently, biometrics recognition have attracted a lot of research and many works are reported in the literature. Biometrics technology is used to identify people from their physical or behavioral characteristics. These characteristics have three major specificities, to define each people, to be stable in the course of time and to be defined without a lot of contraints for users. So, various technologies were based on fingerprints, iris, face, voice, signature, gait or hand. Hand biometrics present many advantages compared to other biometrics technologies. Its characteristics are relatively stable and present a lot of discriminating features such as principal lines, wrinkles, ridges or hand shape. In addition, they present an high user acceptability and the hand can be obtained from low-resolution images with cheaper devices. Many algorithms have been defined for hand authentication based on palmprint [1], [2] or hand-shape recognition [4]. All these algorithms can be decomposed in four stages: the acquisition, the hand segmentation (and palm definition if necessary), the features extraction and the comparison with the reference. Classicaly, the acquisition is a hand scan. Then, in palmprint recognition, the hand is segmented by a fixed threshold and the palm is generally defined from hand shape curvature [2]. Next, various methods have been proposed for recognition. They are Sobel and morphological operations, Fourier spectrum, 2D Gabor phase [2], wavelet signatures, eigenpalm and eigenfingers features, phase and orientation code [7], etc. The last papers present really good results in recognition rate using generally a comparison method based on Hamming distance adjusted following the features. In all these systems, the hand acquisition is in fact the principal

\footnotetext{
J. Doublet and $O$. Lepetit are with France Telecom/Orange R\&D, 42 rue des coutures, 14000 Caen, France julien.doublet@orange-ftgroup.com, olivier.lepetit@orange-ftgroup.com

M. Revenu is with the GREYC-ENSICAEN, Universite de Caen, 6 Boulevard maréchal Juin, 14000 Caen, France Marinette.Revenu@greyc.ensicaen.fr
}

limit. It is nonhygienic, all users touch the same glass, and some artefacts can be created during the acquisition according to the pressure of users on the plate glass.

In this paper, we propose a contactless acquisition with a webcam to reduce these limitations and to get a quick and easy image acquisition. Hand detection process from these acquisitions is a complex task. Many methods are defined for hand detection in man-machine interactions like skin blob tracking, active contours, mean shift or condensation algorithm but provide an approximate detection of each finger. To increase the segmentation quality, we define a method combining information of skin color and hand shape. The segmentation phase is described in section 2. In section 3 , we propose a feature extraction by a robust decomposition and a palmprint matching using a distribution estimation. Section 4 contains some experimental results and section 5 provides the conclusions.

\section{Preprocessing}

Palmprint recognition preprocessing is used to define the central part of the hand (the palm). The most part of these preprocessing algorithms are decomposed in three steps. Firstly the hand is binarized by thresholding. Next, the hand contour is extracted and some key points are defined. Finally, from theses points a coordinate system is defined and the palm is extracted. In our system, the architecture is keept but the methods are adapted for a contactless system.

\section{A. Accurate hand detection}

In biometrics recognition systems, the first step consists on a definition of an accurate and fast segmentation process. In this section, we describe our hand detection method without tracking. This detection uses a fusion of skin color modelling and a robust active shape model.

1) Skin color segmentation: Classicaly the skin color is modelled by Bayes classifiers or Gaussian Mixture Models [8]. Contrary to these methods, our skin color is modelled by machine learning and for a good compromise between the execution time and the precision of detection, we use a neural network (NN). The NN entries are composed by three neurons, one for each color component of pixels in RGB domain. The NN output is the probability that a pixel is a skin pixel. The learning phase permits to model the skin tone in RGB domain. In parallel, a Principal Components Analysis on a skin pixels database defines a specific color space nammed skin space. After the learning, the NN can detect the pixels looking like skin. For each pixel of an image, the NN computes the probability that each pixel is a 


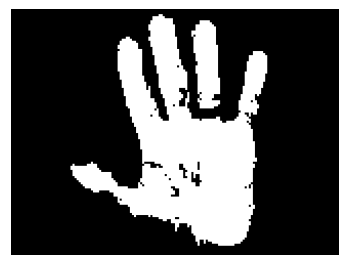

(a)

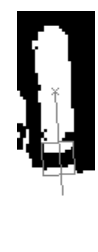

(b)

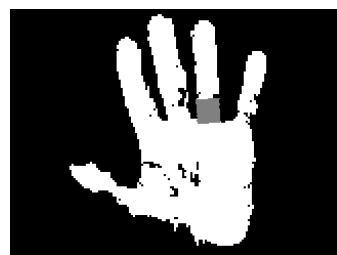

(c)
Fig. 1. Fingers re-connection: (a) binarized map (b) patch adjustment (c) binarized map with patch

skin pixel. This process builds the probabilities map.

To obtain a segmentation close to real time, a multiresolution process builds the probabilities map. This process computes the probabilities map at a lower resolution. Next, the accuracy is increased in computing in better resolution the probabilities that the pixels are skin pixels only if the pixels are near skin and non-skin transition in a lower resolution. Many methods are defined using the probabilies map to clearly identify the detected skin object (hand or face) and to get an accurate segmentation. The principal method consists in segmentation by histogram thresholding on the probabilities map [9], [10]. But more complex processes are defined. Brethes et al. [11] use watershed algorithm, Lui and Izquierdo [12] use median filtering while Tomaz and al. [13] apply a skin pixels grouping. In our approach, a morphological dilatation with a circular element of radius one pixel is carried out on the probabilities map. Next, the probabilities map is segmented by histogram thresholding. This threshold is fixed to 0.5: each pixel is considered as skin color if the neural network output value is more that 0.5 else it is classified as background tone. Finally, as our hand detection process will be used for biometrics recognition, we suppose that only one hand is presented to the system. So the region with the largest surface is preserved and considered as the hand. The regions with an important size and elongation are so conserved. They can be some fingers disconnected from the hand for the users wearing some rings. To reconnect these regions to hand, each region is approximated by an ellipse and we search in the direction of the major axis if the hand is close. If the region is near to the hand, an adjusted patch is added to reconnect the finger to the hand. This patch has a width equals to the width of the minor axis and a length depending on the distance between the finger and the hand. It is oriented with the major axis direction and centred between the hand and the finger. This process is illustrated in Figure 1.

2) Color active shape model : The segmentation by skin color can not carry out the task of hand detection in a robust way. So, a specific active shape model is defined to cancel this problem and to solve the two major difficulties of active shape model [14]. These problems are the contour initialization which must be close to the real form and the model convergence in the detection phase.

Classically, the form to detect is defined by a set of points: the landmarks. In training phase, the average form and the contour variations are computed by Principal Components
Analysis on an annotated hands database by these landmarks. In segmentation phase, the contour is initialized by the characteristic points of the hand: the five fingertip points and the four points located in the valley between two adjacent fingers. These points are calculated from the skin segmentation by distance analysis between a fixed point and each contour points. Next, two other points are automatically added close to wrist from these points. The others landmarks defining with more precision the hand shape are disposed between those. Thus, the model $X$ is obtained by the 11 initial points and $M$ intermediate points disposed between those. $X$ is defined by $[X[0], X[1], \ldots, X[(M+1) \times 10]]$ where $X[i]$ is the ith landmark. After the initialization phase, the model is deformed. To control the problem of model divergence which does not follow the real hand contours, a weight is applied to deformations to limit the shape constraints. In addition, the gradient is computed in the skin color space by Di Zenzo algorithm. Next, this gradient is balanced by the coefficient of the probabilities map pixels. So, the background objects do not pertub the contour evolution. The experiments show that a good compromise between the execution time and the detection precision is obtained by fixing $M$ at 12 . This complete detection process is illustrated on Figure 2(a-d).

\section{B. Palm definition}

After hand detection, it is necessary to extract the acquired palm independently of the distance between the hand and the capture device. Our extraction is based on hand dimensions and the palm extraction method described by Kong and al. [2]. In [2], two values are fixed: the distance between the points $O 1$ and $O 2$ and the palm size $\|A 1 A 2\|$ (Figure 3). These constant values in traditional recognition systems are defined here according to hand sizes. They are determined from the hand width, computed by the Euclidean distance between the points $X[L 1]$ and $X[L 2]$ where $L 1$ and $L 2$ are the indices fixed after experiments at 30 and 125 . Thus,

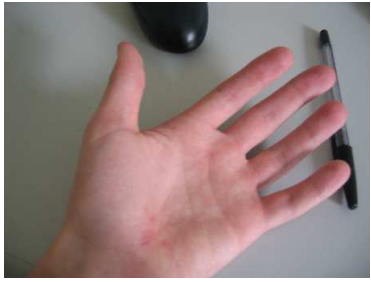

(a)

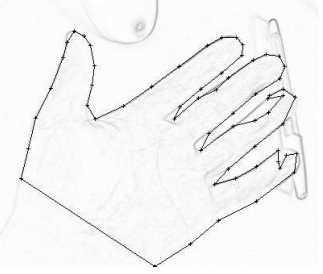

(c)

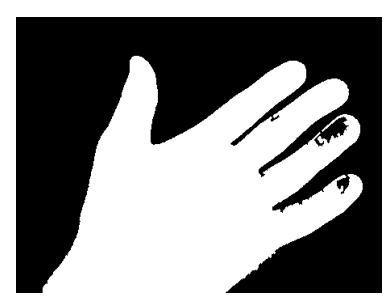

(b)

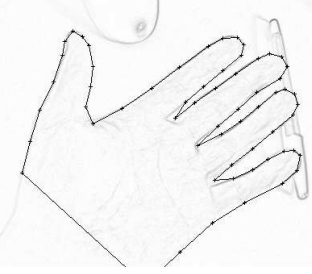

(d)
Fig. 2. Hand detection process: (a) original image (b) skin segmentation (c) initial hand shape (d) final hand shape 


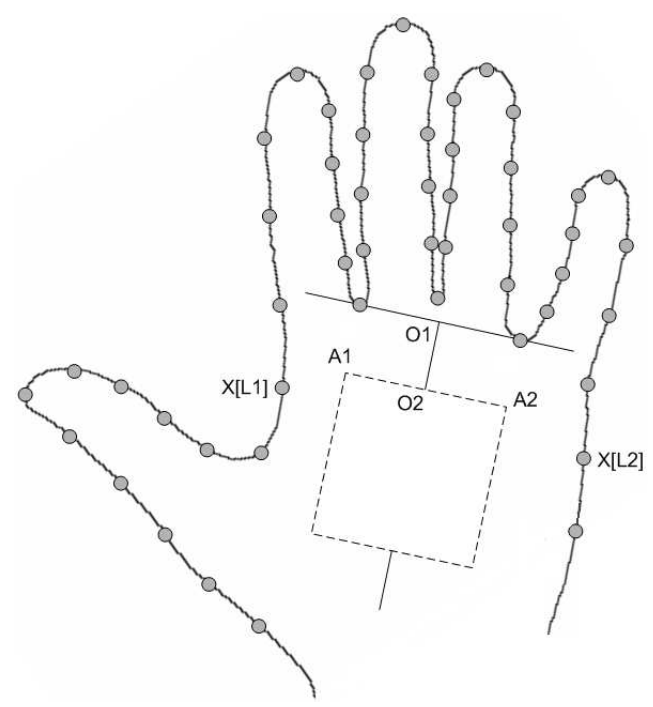

Fig. 3. Schematic palm extraction with $M=4$

||O1O2|| and $\|A 1 A 2\|$ are defined by:

$$
\left\{\begin{aligned}
\|O 1 O 2\| & =\alpha\|X[L 1] X[L 2]\| \\
\|A 1 A 2\| & =\beta\|X[L 1] X[L 2]\|
\end{aligned}\right.
$$

Where $\alpha$ and $\beta$ are the dimension coefficients chosen at $1 / 10$ and $2 / 3$, respectively. Next, the palm is resized with a fixed size $N \times N$.

The extracted palm contains some principal lines which can be determined by a specific palm line extraction. These lines are not unique to each individual, thus it is necessary to use the ridges and the secondary lines of the palm. This information cannot be extracted from the palm with images in low resolution, so a global palm characterization is suitable.

\section{ROBUST FEATURES EXTRACTION}

From preprocessing, the palm features must be extracted. A lot of method have been proposed to define the specific information of each user. The most important methods consist of statistical approach (decomposition into small regions and computation of the means and the variance of each region), subspace-based approach (Principal Component Analysis, Independent Component Analysis or Discrete Cosine Transform) and filtering approach (encoding filter responses). In all these systems the noise in images are generally not taken into consideration as the acquisition is performed in a constraint environment and with an adequate device.

In our system the sensor is limited (webcam or camera phone). So we proposed to limit the noise by a PDE (Partial Differential equations) regularization. Moreover in the majority of statistical systems and subspace-based approaches, the luminosity changes are managed by a global histogram equalization or a normalisation of the palm to a fixed average and variance. As these methods are not robust to inhomogeneous luminosity changes, we proposed a normalisation with a palm decomposition. These two methods are described in the following paragraphs.

\section{A. Palm restoration}

The images acquire with low quality devices present important noises (typically following a poisson distribution) and compression artefacts specially in an environment with difficult lighting conditions. These two types of pertubations are difficult to remove and many methods tends to resolve this problem. A well tool to make a powerfull regularisation of noisy images is the anisotropic diffusion PDE's. PDE regularisations are based on locale image smoothing. This smoothing is depending on the gradient information: with important gradient, the smoothing is effectued along the gradient direction else, in homogeneous region, the smoothing is performed in all directions. So, these methods give an anisotropic regularisation without edges destruction. Recently, Tschumperlé proposed an anisotropic smoothing of images using curvature-preserving PDE's fast and maintening the thin image details [18]. This method is based on a separation of the smoothing geometry from the smoothing process respecting the local smoothing geometry. Moreover, the author defines a regularisation given into consideration the curvature of the image contour. The complete description of this algorithm is done in [18]. A regularisation example on a low-qualtity palm is showned in Figure 4. It's important to note that this regularisation is applied only in palm restoration and not for the total image restoration to limit the computing time. In fact, the multi-resolution computation in skin detection process and the hand shape learning limit the noise impact in the hand detection process.

\section{B. Palm decomposition and normalisation}

From the restored palm, we propose to make a local normalisation to be robust of inhomogenous luminosity change. Let $I_{l}$ an image $I$ with an additional uncontrolled luminosity impact and $I(x, y)$ the gray level at the point $(x, y) . I_{l}(x, y)$ can be defined by:

$$
I_{l}(x, y)=I(x, y)+L(x, y)
$$

where $L(x, y)$ defined the luminosity impact at the point $(x, y)$. An approximation perhaps carried out by taking into account the fact that close points have an identical luminosity factor. So, the definition (2) is redefined by:

$$
I_{l}(x, y)=I(x, y)+L_{r}(x, y)
$$

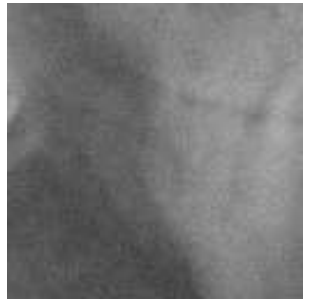

(a)

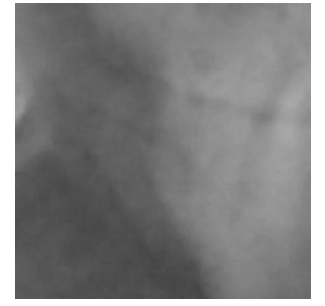

(b)
Fig. 4. A restoration example of a noisy palm image: (a) input palm (b) restored palm 

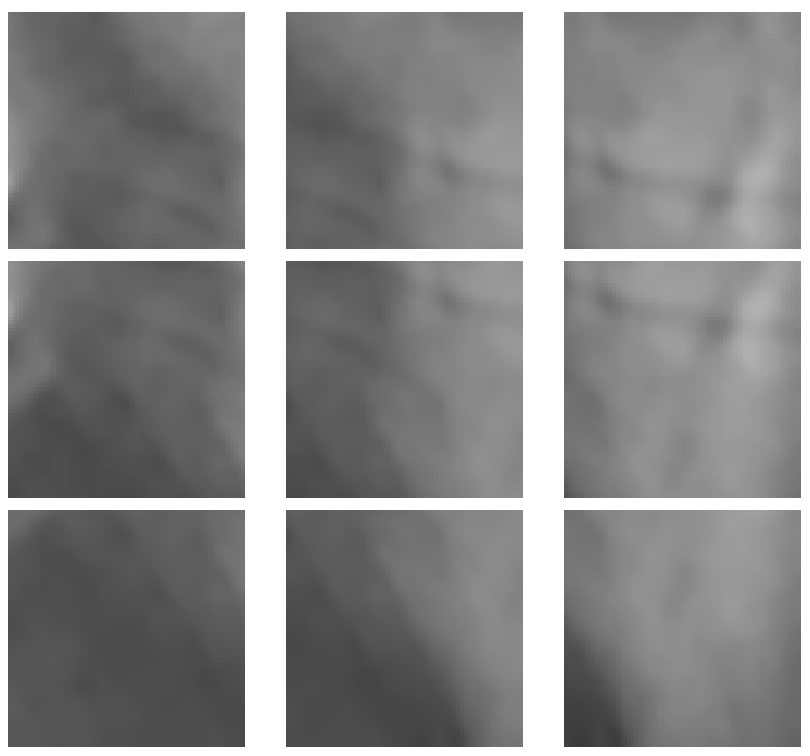

Fig. 5. Decomposition and normalisation of the restored palm image of the Figure 4

where $L_{r}(x, y)$ is the luminosity impact in a region near the point $(x, y)$. Using this definition, we proposed to normalized the palm image by a decomposition of this image into adjacent small regions that are supposed to have the same luminosity impact and a standardization of these regions.

Firstly a palm image is decomposed into $\Lambda$ regions defining a set of sub-images $\left\{R_{0}, R_{1}, \cdots, R_{\Lambda}\right\}$. The number of regions depends on the sub-image size $S \times S$ and the intersection width between each region $W$.

Next, each region $R_{i}$ is normalized to a prespecified mean and variance. The mean $\phi_{i}$ and the variance $\rho_{i}$ of each region $R_{i}$ are computed before standardization. With these data, each normalized region $R_{i}^{\prime}$ is determined by:

$$
R_{i}^{\prime}= \begin{cases}\phi_{i}^{d}+\lambda(x, y) & \text { if } R_{i}(x, y)>\phi_{i} \\ \phi_{i}^{d}-\lambda(x, y) & \text { otherwise }\end{cases}
$$

where

$$
\lambda(x, y)=\sqrt{\frac{\rho_{i}^{d}\left(R_{i}(x, y)-\phi_{i}\right)^{2}}{\rho_{i}}}
$$

and $\phi_{i}^{d}$ and $\rho_{i}^{d}$ are the desired mean and variance of the region $R_{i}$, respectively. So, the palmprint feature $R^{\prime}$ is a set of normalized sub-image $\left\{R_{0}^{\prime}, R_{1}^{\prime}, \cdots, R_{\Lambda}^{\prime}\right\}$.

As each user has different palms, the desired data (mean and variance) are defined individualy for each user to keep the most possible information. In a learning phase, these specific values are fixed by the medium value of variance and mean of palmprint regions for each user. Figure 5 shows an illustration of decomposition and normalisation process applied to the restored palm of Figure 4 with the parameters $S=N / 2$ and $W=N / 4$.

\section{REFERENCE DEFINITION BY DISTRIBUTION ESTIMATION}

From the palmprint features, a decision function must be defined to verify if two references are similar. We propose to use a classification method. With its good accuracy, we used an estimation of the support of a high-distribution estimation [19] based on the Support Vector Machine (SVM) introduced by Vapnik [20].

\section{A. Distribution estimation}

Initialy, the SVM permits to classify some elements in different classes with linear functions by projecting the data in a higher dimensional space. In [19], Scholkopf and al. proposed an extension of the support vector algorithm in the case of unlabeled data in estimating the support of a high-dimensional distribution.

Given a training data $\left\{x_{1}, \cdots, x_{l}\right\}, i=1, \cdots, l$ where $l$ is the number of exemples, $x_{i} \in \mathbb{R}^{m}$ is the exemple of index $i$ and $m \in \mathbb{N}$ is the data dimension. Let $\Phi$ a function of projection $\mathbb{R}^{m} \rightarrow F$, a kernel function $K\left(x_{i}, x_{j}\right)=$ $\left(\Phi\left(x_{i}\right) . \Phi\left(x_{j}\right)\right)$ is defined. An exemple of the kernel function is the radial basis function defined by:

$$
K\left(x_{i}, x_{j}\right)=\exp ^{-\gamma\left\|x_{i}-x_{j}\right\|^{2}}, \gamma>0
$$

The goal of the method proposed by Scholkopf and al. is to determine a function $f$ that return +1 in a region near the training data and -1 elsewhere. The objective is to map the data in a feature space and to separate them with maximum margin. The data set can be separated from the origin in solving the following primal problem:

$$
\begin{array}{r}
\min _{w, \xi, \rho} \frac{1}{2} w^{T} w+\frac{1}{v l} \sum_{i=1}^{l} \xi_{i}-\rho \\
\text { subject to } w^{T} \Phi\left(x_{i}\right) \geq \rho-\xi_{i}, \xi_{i} \geq 0 .
\end{array}
$$

where $\left.\left.\xi \in \mathbb{R}^{l}, \rho \in l, w \in F, v \in\right] 0,1\right]$ controls the number of support vectors and the errors. $v$ is an upper bound on the fraction of the outliers (training points outside the defined region) and a lower bound of the fraction of support vectors. The dual problem of (6) can be obtained in introducing a Lagrangian. This dual can be explained by:

$$
\min _{\alpha} \frac{1}{2} \sum_{i j} \alpha_{i} \alpha_{j} K\left(x_{i}, x_{j}\right)
$$

$$
\text { subject to } 0 \leq \alpha_{i} \leq \frac{1}{v l}, \sum_{i=1}^{l} \alpha_{i}=1 \text {. }
$$

where $\alpha_{i} \geq 0$. Using the Lagrangian, the decision function $f$ can be explained with the kernel function by:

$$
\begin{gathered}
f(x)=\operatorname{sign}\left(\sum_{i=1}^{l} \alpha_{i} K\left(x_{i}, x\right)-\rho\right) \\
\text { where } \operatorname{sign}(a)= \begin{cases}+1 & \text { if } a \geq t \\
-1 & \text { otherwise }\end{cases}
\end{gathered}
$$


Classicaly, the threshold $t$ determining the decision is fixed to the value 0 .

\section{B. Individual Authentification function}

In our palmprint matching, we propose to use a decision function for each user. So, in learning phase a decision function $f_{I_{d}}$ is estimated for each client $I_{d}$. The palmprint features of each user $R_{I_{d}}^{\prime}$ defined in section III are transformed into a $1 \mathrm{D}$ vector $X_{I_{d}}$ by concatenation each element of the reference $R_{I_{d}}^{\prime}$ row by row. $\Gamma$ references $X_{I_{d}}^{j}, j=$ $1, \cdots, \Gamma$ defined of class +1 are used as training data to defined this estimation. Because the palm extraction process is imperfect, each palmprint feature used to define the decision function is translated (vertically and horizontally) and rotated to supplement the training data. The ranges of the translation and the rotation are defined from -2 to 2 pixels and -2 to $2^{\circ}$ respectively.

The learning phase is fast with so few exemples but it's not possible to determine a same fixed thresholding $t$ for all users to separate impostor and genuine because the decision function is only depending on a set of positive examples. So, for each user $I_{d}$ a threshold $t_{I_{d}}$ must be defined. This threshold $t_{I_{d}}$ is computed in projecting the references $X_{I_{d}}^{j}, j=1, \cdots, \Gamma$ into the function $f_{I_{d}}$. Next, the medium value of these projections is kept and adjusted with a value $\tau$ to define the threshold. This definition can be explained by:

$$
t_{I_{d}}=\operatorname{medium}_{j}\left(f_{I_{d}}\left(X_{I_{d}}^{j}\right)\right)-\tau
$$

The parameter $\tau$ limits the impact of bad palm extraction and noisy images in matching phase. The medium value is used to get the notion of variation between a genuine palmprint feature and the decision function and to be robust to a training feature obtained with an important bad palm acquisition.

\section{EXPERIMENTATIONS}

A specific database is made to validate our approach. All images of the database were acquired by a webcam Philips ToUcam Pro $740 \mathrm{~K}$ with a size of $640 \times 480$. The database contains 490 images of hands, some of these presenting hands with rings, coming from 49 people. Ten images are acquired for each individual. The capture restrictions are that the users must present their hands in front of the camera with theirs fingers separated. The palm is extracted by our hand detection process and the palm is resized to $128 \times 128$ $(N=128)$. Figure 6 shows two examples of hand images with corresponding palms.

Let $X_{I_{d}}^{j}$ the palmprint feature of the palm $j$ of the user $I_{d}$. First, each user $I_{d}$ is described by a function decision $f_{I_{d}}$. The training data for each user to fixe this function and the variances and means which permit the normalisation of the decomposed palm are defined by the set $\left\{X_{I_{d}}^{j}\right\}(j=$ $1, \cdots, \Gamma)$ of class +1 . In evaluation phase, a test database is defined for each user. This database is explained for an identity $I_{d}$ by a genuine set $\left\{X_{I_{d}}^{j}\right\}(j=\Gamma+1, \cdots, 10)$ of
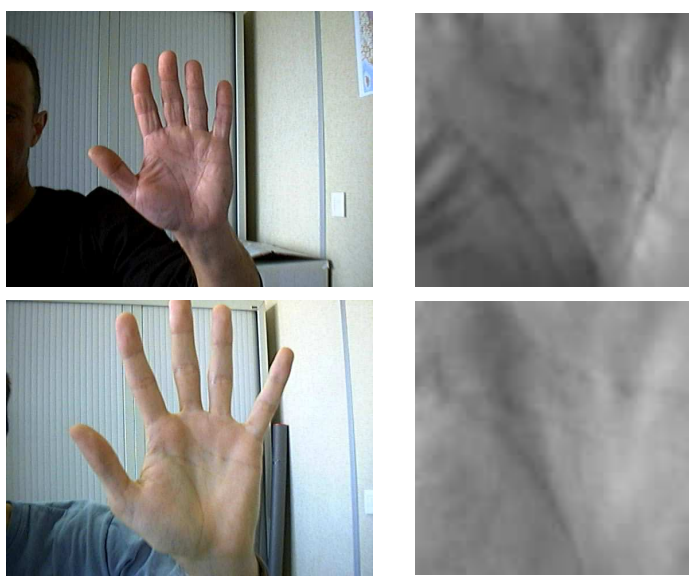

Fig. 6. Two examples in our database and corresponding palms

class +1 and an impostor set $\left\{X_{I_{e}}^{j}\right\}(e=1, \cdots, 49$ and $e \neq$ $d, j=1, \cdots, 10)$ of class -1 . All these data are standardized by the means and the variances defined in learning phase. To increased the number of test, the complete process of experimentations (learning and evaluation) is done ten times in randomly changed the index $j$ of each reference.

In the complete evaluation phase, the rates of classification are determined. A comparison is incorrect if a user is accepted or rejected wrongly. Two rates characterize the accuracy of the system: the false acceptance rate $(F A R)$ defined by the ratio of people authenticated wrongly and the false rejection rate $(F R R)$ defined by the ratio of people rejected wrongly. All recognition rates presented in this paper correspond to 100 - equal error rate $(E E R)$ defined when $F A R=F R R$.

Different values for the system parameters $\Gamma, K$ and $\tau$ are used to obtain the best recognition rate. The parameter $\Gamma$ explain the number of exemples to form training set. When the threshold adjusted by $\tau$ varies the recognition rates are different. Table I shows the system performance following these two datas. It is found that the recognition rate is higher then the size of the training data increased. With a parameter $\tau$ optimally fixed to 0.04 , the recognition rate is $98.5 \%$ with seven training examples. The authentication process are computed with a kernel $K$ of type radial basis function. Moreover, the selection of the Kernel function is a difficult and on open problem. So, an experiment is done to establish the Kernel given the best representation of the data. The experiment results with linear, polynomial and radial basis functions are showed in Figure 7 and indicate that the radial basis function is optimal in our system.

The complete process of recognition (segmentation, characteristics extraction and matching) is carried out in less than 1 second on Pentium M with $1.60 \mathrm{GHz}$. The enrollement time is depending on the number of training exemples. With $\Gamma=5$, the decision function computation is done in 4.7 seconds. 
TABLE I

COMPARE THE PERFORMANCE OF THE SYSTEM FOLLOWING THE NUMBER OF TRAINING SAMPLES AND THE ADJUSTMENT THRESHOLD $\tau$

\begin{tabular}{llllll}
\hline \hline$\tau$ & $\Gamma=3$ & $\Gamma=4$ & $\Gamma=5$ & $\Gamma=6$ & $\Gamma=7$ \\
\hline & & & & & \\
0.02 & $81.5 \%$ & $83.5 \%$ & $84.7 \%$ & $85.8 \%$ & $86.1 \%$ \\
0.03 & $85.2 \%$ & $88.1 \%$ & $92.5 \%$ & $93.4 \%$ & $93.6 \%$ \\
0.04 & $92.5 \%$ & $94.9 \%$ & $96.2 \%$ & $97.0 \%$ & $97.3 \%$ \\
0.05 & $93.7 \%$ & $96.0 \%$ & $97.3 \%$ & $98.4 \%$ & $98.5 \%$ \\
0.06 & $88.1 \%$ & $92.5 \%$ & $92.7 \%$ & $93.3 \%$ & $93.6 \%$ \\
0.07 & $83.2 \%$ & $84.2 \%$ & $85.9 \%$ & $86.3 \%$ & $86.4 \%$ \\
\hline \hline
\end{tabular}

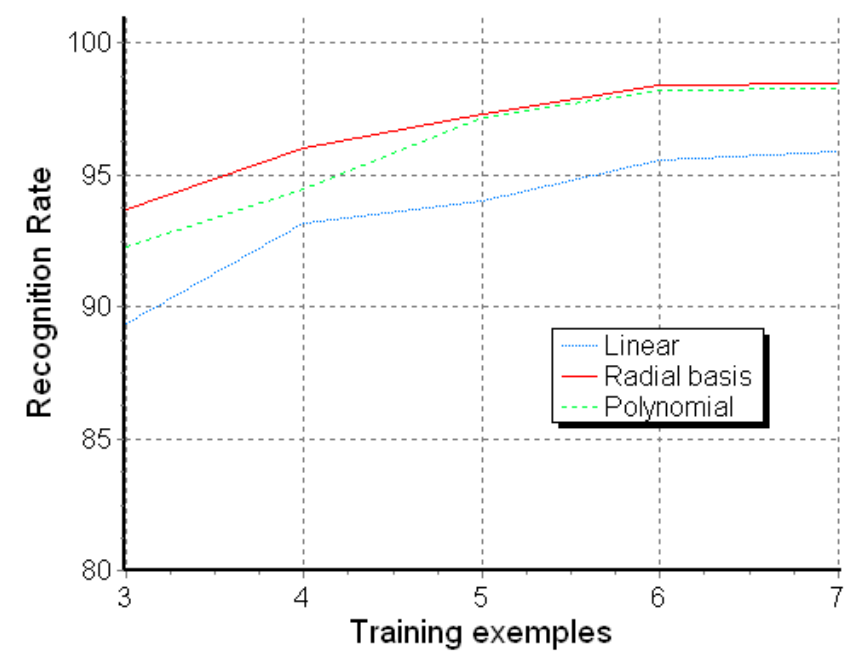

Fig. 7. Recognition rates with different Kernels

\section{CONCLUSIONS}

In this paper, we present a new method of biometrics hand recognition for a contactless system. First of all, the hand segmentation is explained. It is carried out thanks to an integration of the skin color components and a shape model. Then, the authentication process based on robust features extraction and a specific distribution estimation is exposed. The features extraction permits to get an information robust to illumination change and limited noise. Next, an individual threshold is defined for each specific decision function. The complete process is validated after experiments on a database of 49 people. It shows an error rate of $1.5 \%$ with an execution time lower than 1 second.

In order to manage space rotations of the hand, an invariant method with the perspective and the shear will have to be required. Moreover, a definition of the value $\tau$ for each user must be defined. These two tracks are currently the subject of our work.

\section{REFERENCES}

[1] N. Duta, A. Jain and K. Mardia: Matching of palmprint, Pattern Recognition Letters 23 (4) (2001) 477-485.

[2] W. Kong, D. Zhang and W. Li: Palmprint feature extraction using 2-d Gabor filters, Pattern Recognition 36 (2003) 2339-2347.

[3] X. Wu, K. Wang, D. Zhang: Wavelet Based Palmprint Recognition, Conference on Machine Learning and Cybernetics, Beijing (2002).
[4] E. Yoruk, E. Konukoglu, B. Sankur and J. Darbon: Shape-Based Hand Recognition, IEEE Trans. on Image Proc. 15 (7) (2006) 1803-1815.

[5] A. Kumar, C. Wong, C. Shen and A. Jain: Personal Verification using palmprint and geometry biometric, Int. Conf. on audio and video based biometric person authentication, Guilford (2003).

[6] S. Ribaric, I. Fratric: A Biometric Identification System Based on Eigenpalm and Eigenfinger Features, IEEE Trans. Pat. Anal. Mach. Intell. 27 (11) (2005) 1698-1709.

[7] X. Wu, K. Wang, F. Zhang and D. Zhang: Fusion of Phase And Orientation Information for Palmprint Authentication, Pat. Anal. and App. 9 (3)(2006) 103-111.

[8] V. Vezhnevets, V. Sazonov et A. Andreeva: A survey on pixel-based skin color detection techniques, (2003).

[9] S. Sural, G. Qian and S. Pramanik: Segmentation and histogram generation using the HSV color space for image retrieval, Int. Conf. on Color Imaging: Proc., Hardcopy, and Applications, San Jose, USA (2005).

[10] S. Singh, D. Chauhan, M. Vatsa and R. Singh: A robust skin color based face detection algorithm, Int. Tamkang journal of Sc. and Eng. 6 (4) (2003)

[11] L. Brethes, P. Menezes, F. Lerasle, J. Hayet: Face tracking and hand gesture recognition for human-robot interaction, ICRA04, New Orleans, USA (2004).

[12] T. Lui and E. Izquierdo: Automatic Detection of Human Faces in Natural Scene Images by Use of Skin Color and Edge Distribution, 5th Int. Workshop on Image Analysis for Multimedia Interactive Services, Lisbon, Portugal (2004)

[13] F. Tomaz, T. Candeias and H. Shahbazkia: Improved Automatic Skin Detection in Color Images, Int. Conf. on Digital Image Computing: Techniques and Applications, Sydney, Australia (2003).

[14] T. Cootes and C. Taylor: Statistical models of appareance for computer vision, Technical report, University of Manchester, UK (1999).

[15] J. Zhang, T. Tan and L. Ma: Invariant Texture Segmentation Via Circular Gabor Filters, ICPR02 2 (2002) 901-904.

[16] T. Hoad: Video representations for effective retrieval from large collections, PhD thesis, Department of Computer Science and Information Technology, RMIT University (2004).

[17] J. Daugman: High Confidence Visual Recognition of Persons by a Test of Statistical Independence, IEEE Trans. Pattern Anal. Mach. Intell. 15 (11) (1993) 1148-1161.

[18] D. Tschumperlé: Fast Anisotropic Smoothing of Multi-Valued Images using Curvature-Preserving PDE's, International Journal of Computer Vision, IJCV(68), No 1, June 2006, pp.65-82.

[19] B. Scholkopf, J.C. Platt, J. Shawe-Taylor, A.J. Smola and R.C. Williamson:Estimating the support of a high-dimensional distribution, Neural Computation, 13, 7, 1443-1471, 2001.

[20] V. Vapnik: Statistical Learning Theory, Wiley, 1998. 